\title{
Recherche intitulée \\ " Le personnage romanesque dans \\ Le sermon sur la chute de Rome \\ de Jérôme Ferrari: du héros à l'anti-héros"
}

\author{
Présentée par: \\ Dr. Nader Anwar Hénawi \\ (Maître de conférences \\ département de français \\ Faculté Al Alsun \\ Université d'Ain Chams)
}

\section{Introduction.}

$\mathrm{Si}$ les romanciers ont toujours fait de leurs personnages des héros mythiques qui luttaient et combattaient en faveur d'un idéal, l'auteur du XXe et du XXIe siècles continuera-t-il dans la même voie destinée à idéaliser ses personnages ou à les doter d'une positivité absolue? À un siècle où se multiplient les guerres et les génocides et où l'on prononce des discours philosophiques sur le mal, le néant et l'absurde, le personnage romanesque demeurera-t-il le même être qui possède un caractère sublime et poétique, qui fait preuve de stoïcisme et de courage et qui lutte hérö̈quement contre l'adversité? Ce type ne place-t-il pas le romancier dans une tour d'ivoire et ne fait-il pas, en même temps, de la littérature un discours chimérique? À cet égard, certains critiques littéraires affirment que:

"(...) l'art est avant tout arbitraire, il réorganise, retranscrit, modifie, il projette le personnage dans un univers de l'absence au monde, faisant de la créature romanesque un être de l'altérité où le monde réel étiolé devient espace de l'étoile, planète imaginaire. " (MIRAUX, 1997: 66)

En effet, le monde actuel est le théâtre de nombreux accidents historiques: conflits mondiaux, affrontements de classes, montée des extrémismes, mercantilisme généralisé etc. Par conséquent, la littérature (qui est censée refléter le réel) doit être nimbée de pessimisme et d'incertitude et doit mettre en scène des personnages qui subissent les coups du destin et qui s'avèrent incapables d'agir ou d'avoir une prise concrète sur le monde.

Né en 1968, Jérôme Ferrari est parmi les écrivains qui ont souligné l'impact des bouleversements du monde sur le personnage. Cet homme a effectué une partie de ses études à la Sorbonne, où il a obtenu la licence de philosophie de l'université Paris 1 Panthéon-Sorbonne. Il a vécu en Corse et a 
enseigné la philosophie au lycée de Porto-Vecchio. Il a obtenu le prix Goncourt 2012 pour son livre Le sermon sur la chute de Rome, objet de notre étude.

Dans ce roman, l'écrivain relate l'histoire de Marcel Antonetti, un homme à la santé fragile dès son enfance. En dépit de son état lamentable, Marcel résiste stoïquement à sa maladie qui freine sa démarche vers le progrès et vers l'absolu. Avec le temps, Marcel succombe à son ulcère gastralgique qui acquiert un contour de plus en plus grave. En effet, il s'assure qu'il est incapable de lutter contre le destin. Le récit accorde également une place majeure à Matthieu (le petit-fils de Marcel) qui a abandonné ses études philosophiques pour gérer (en compagnie de son ami Libero) un café ou un bar en Corse, où habitait autrefois son grand-père (Marcel Antonetti). Ce bar devient le théâtre de plusieurs mutations diversifiées: succès, échecs, déceptions, concurrences, plaisirs, malheurs etc.

Dans cette recherche, nous analyserons la figure archétypale de l'« anti - héros ». Personnage principal de l'oeuvre, ce nouveau héros est doté de tous les aspects négatifs. Il est dépourvu de toutes les qualités traditionnelles qui font de lui un être digne de respect. Passif et paralysé, il est marginalisé au sein de la foule et dispose de tous les défauts qui le rendent un être vulnérable et vaincu. Il est sans épaisseur psychologique, sans identité. Sa présence au sein de la foule ne fait qu'accentuer sa problématique, comme en témoigne Michel Erman:

"En général, un anti-héros est plongé dans le flux de la vie mais il se heurte aux autres et ne pèse guère sur les événements comme s'il considérait l'existence à partir de l'absence et avait peine à s'inscrire dans le temps. " (ERMAN, $2006: 117)$

Terne, il ne fait que subir les aléas de la vie quotidienne, déréglée et labyrinthique. Il ne fait aucun exploit, se contentant de vivre au jour le jour, sans rien attendre de la vie car il ne possède ni présent ni avenir. Résigné, il ne cherche pas à faire des projets. Il vit dans des lieux mornes qui accentuent son affliction et où personne ne l'attend ni ne l'apprécie vraiment. N'ayant pas la liberté d'agir, il se voit incapable de transformer ses désirs en actes concrets. Son identité défectueuse et sa personnalité inconsistante l'invitent à éprouver des sentiments contradictoires allant de la souffrance à l'épicurisme, de l'obsession par les idées morbides à la quête des plaisirs.

Si nous avons choisi Le sermon sur la chute de Rome comme objet d'étude, c'est parce que ce roman revêt un aspect réaliste et vraisemblable. En effet, il met en scène des univers contrastés (le bien-le mal/ le stoïcisme-l'épicurisme/ l'action positive- la paresse/ la résistance-la résignation); autant de contradictions incarnées par tout être humain qui se voit tiraillé entre plusieurs états antithétiques, qui ignore sa propre voie dans ce monde aléatoire et labyrinthique et qui devient incapable de se fixer des priorités et 
des buts idéaux au sein d'un univers chaotique et précaire, au sein d'un monde qui le déçoit et qui ne lui apporte qu'angoisse, malheur et humiliation. En effet, le roman de Ferrari décrit des êtres égarés et marginalisés, des personnages errants qui tâtonnent dans les ténèbres. Ainsi le lecteur s'identifie-t-il à toutes ces victimes et devient-il familiarisé avec leurs crises et leurs souffrances.

Notre problématique consiste alors à étudier le lien étroit entre l'image de l'« anti-héros » et l'aspect réaliste de la littérature, c'est-à dire entre le vécu et le raconté, entre le monde et sa représentation. Notre objectif tend à dévoiler comment l' "anti-héros »s'est imposé au roman moderne et a réussi à éliminer le héros classique, noble, honnête et sublime, comment il est devenu le miroir dans lequel le lecteur contemporain découvrait son visage. En d'autres termes, nous voulons prouver comment la déconstruction du personnage mène à la reconstruction du roman, comment cet " anti-héros " jouit d'une crédibilité et d'une profonde vraisemblance, et ce contrairement au héros traditionnel, cette figure qui possède des qualités chevaleresques, mais qui, toutefois, n'a pas de véritable place dans notre monde réel, provoquant non seulement le discrédit de l'art romanesque mais aussi le dénigrement de la littérature, en général.

Quant à la méthode que nous emploierons, nous adopterons l'approche « dialectique ». Nous parlerons tout d'abord de l'état de Marcel en tant que personnage stoïque qui refuse de succomber à la maladie et qui résiste aux malheurs, puis nous évoquerons l'autre côté opposé de ce personnage en énumérant les divers aspects de sa médiocrité. Une telle malédiction qui se transmet de Marcel (le grand-père) à Matthieu (le petit-fils), comme si le portrait de l'anti-héros était devenu le type qui caractérise tous les romans du XXIe siècle. La méthode dialectique nous permettra donc de parler d'une thèse (la lutte épique d'un héros positif) et de la réfuter en examinant son antithèse (l'échec du personnage qui se transforme en anti-héros).

En parlant de l'« anti-héros ", nous nous sommes fondé sur quelques concepts théoriques publiés par Pierre Glaudes dans son livre intitulé " Personnage et Histoire Littéraire » (1991) et par Francis Berthelot dans son ouvrage intitulé "Le corps du héros. Pour une sémiologie de l'incarnation romanesque » (1997). Tous deux insistent sur la faiblesse physique de l'antihéros ainsi que sur son profil psychologique et moral. Ils évoquent la problématique d'un personnage qui éprouve une peur constante de la dépossession et qui cherche en vain à échapper à la vie commune et routinière ainsi qu'à l'ennui en se détachant du présent. Cet anti-héros essaie également de fuir le regard bourreau des Autres.

Nous tenterons de voir comment Jérôme Ferrari préfère l'homme réel à l'homme fabuleux, l'acte quotidien à l'action extraordinaire, l'être vraisemblable à l'homme aux sentiments factices. En fait, au lieu d'incarner 
un sens péjoratif et négatif ou de signaler la défaite de l'art romanesque, l'« anti-héros » enrichit la littérature et lui permet de refléter les chaos du monde actuel, banal, prosaïque et périssable. L'auteur du Sermon sur la chute de Rome parviendra-t-il à faire de l'anti-héros le meilleur reflet des incohérences et des aléas de la vie quotidienne? Réussira-t-il à sortir l' " anti-héros » du moule qui le fige toujours dans une situation subalterne? Parviendrons-nous à défendre notre point de vue? C'est ce que nous essaierons de voir dans cette recherche.

\section{La lutte épique d'un héros "positif».}

Dès le début, Le sermon sur la chute de Rome est considéré comme un hymne au succès, à la gloire et à l'aspect positif de l'action humaine. Le roman s'ouvre sur une situation où l'on trouve que Marcel Antonetti est un héros qui résiste stoïquement à la maladie et aux crises sanitaires dont il souffre dès son enfance:

"Un démon rôdait sans cesse autour de lui, dont ses parents redoutaient la victoire, mais Marcel savait qu'il ne vaincrait pas, il aurait beau le jeter sans forces au fond de son lit, l'épuiser de migraines et de diarrhées, il ne vaincrait pas, il pouvait même s'installer en lui pour y allumer les feux de l'ulcère et le faire cracher du sang avec une telle violence que Marcel dut manquer une année entière d'école, il ne vaincrait pas (...). » (FERRARI, 2012: 16)

Les premières lignes du roman saluent alors les efforts de l'être humain, lequel aspire à l'absolu, au salut et à l'épanouissement et proscrit de son dictionnaire les termes "échec", "défaite" et "chute". Grâce à la répétition de la phrase négative "il ne vaincrait pas », le narrateur neutralise l'effet néfaste de ce démon (l'ulcère gastralgique dont souffre Marcel) et dévoile le triomphe de ce dernier sur le destin, perçu toujours comme une force invincible. Si les symptômes de la maladie témoignent de l'épuisement du personnage et si la crise sanitaire retarde la rentrée scolaire de Marcel, celuici demeure encore vivant et apte à résister au mal et au malheur.

C'est ainsi que la maladie et la souffrance physique nous montrent si nous avons égard à un héros fort ou faible, stoïque ou épicurien, résistant ou chétif. Le héros du roman, objet d'étude, passe donc pour un être robuste, fort et courageux qui refuse d'être une marionnette dans les mains du destin, même s'il possède une vie qui pourrait bien lui être reprise à n'importe quel moment, une vie " constamment menacée et constamment triomphante 》 (FERRARI, 2012: 16). Les quelques lignes ci-dessous nous montrent que la fin de Marcel demeurera à jamais ajournée:

"L'aube n'annonçait qu'un nouveau sursis et Marcel partait vers l'école, s'arrêtant parfois en chemin pour vomir du sang en se promettant de ne rien dire à sa mère qui l'obligerait à se coucher et 
prierait agenouillée à ses côtés en lui appliquant des compresses brûlantes sur le ventre, il ne voulait plus permettre que son démon l'arrache aux seules choses qui faisaient sa joie, les leçons du maître, les cartes de la géographie colorées et la majesté de l'histoire (...). » (FERRARI, 2012: 18)

À chaque jour qui se lève, une nouvelle chance est accordée à l'enfant Marcel pour survivre. L'originalité de ce personnage réside alors dans son caractère sérieux qui l'empêche de se plaindre à ses parents ou de mettre à nu ses souffrances. Il sait bien que les jérémiades ne mèneront à rien et que les Autres seront incapables de l'aider. Ils éprouveront seulement un peu de sympathie à son égard et prendront quelques précautions inutiles destinées à freiner son progrès scolaire et intellectuel au lieu de remédier à son état chronique. Plutôt que de susciter l'aversion du lecteur, la sensation visuelle (due au sang répandu dans le passage ci-dessus) provoque l'admiration du récepteur qui se voit obligé de louer cet enfant. Celui-ci fait preuve de force, refuse de se reposer ou de faire l'école buissonnière et insiste pour continuer ses études.

Marcel est donc décrit non comme un enfant ordinaire mais comme un enfant prodige qui lutte contre la malédiction physique dont il est l'objet mais aussi contre les chocs qu'il reçoit en ayant un contact avec la nature:

" (...) même l'eau des fleuves est trouble et, sur les rivages déserts, le clapotis des vagues exhale un écoeurant parfum de marais, il faut lutter pour ne pas devenir inerte soi-même et se laisser lentement engloutir comme par des sables mouvants, et Marcel mène encore un combat incessant contre les forces déchaînées de son propre corps, contre le démon qui s'acharne à le clouer au lit, la bouche pleine d'aphtes, la langue rongée par le flux des sucs acides, comme si une vrille avait creusé dans sa poitrine et dans son ventre un puits de chair à vif, (...). » (FERRARI, 2012: 67)

À la douleur de l'ulcère qui touche son appareil digestif, s'ajoute un choc dû à l'ensemble des sensations douloureuses et désagréables: visuelle due à l'eau trouble des fleuves, olfactive provoquée par l'odeur nauséabonde des marais, tactile due à la comparaison de son état avec le sable mouvant et gustative provoquée par le flux des sucs acides. Tout afflige donc Marcel, l'ennuie et conspire à lui nuire. Même la nature s'accorde à son tourment intérieur et à son état d'âme. C'est ainsi que nous avons égard à «un dialogue muet d'un personnage et d'un décor, qui se prêtent mutuellement leurs caractères. " (MITTERAND, 1980: 61). Cependant, cette nature dysphorique et cette condition médiocre n'ont pas réussi à détourner Marcel de son but idéal (la résistance contre la maladie ou contre la mort): 
" De l'autre côté de la mer, le démon de l'ulcère semble paralysé, privé de ses capacités de nuisance, et pour la première fois de sa vie, Marcel jouit d'une vitalité dont il ne soupçonnait pas l'existence, il se comporte comme le bon élève qu'il a toujours été et il est sourd à tout le reste (...) » (FERRARI, 2012: 72)

Si Marcel demeure " sourd à tout le reste », c'est parce qu'il s'est créé un monde indépendant vis-à-vis des Autres. Il refusera de voir la sympathie dans le regard de ses semblables car il ne se prend pas pour un faible. Ni la maladie ni la fatigue ne compteront pour lui. Ce qui compte pour lui, c'est de se forger un moi souverain, même s'il ne triomphe pas totalement de sa maladie. Cette attitude positive a été saluée par Francis Berthelot:

"L'une des caractéristiques du héros avec un grand H est en effet, non pas de sortir vainqueur de tous les combats, mais de faire preuve, face à la douleur, d'une volonté plus forte. " (BERTHELOT, 1997: 122)

En effet, Marcel ignore la paresse. Pour lui, le combat est un honneur en soi, même s'il ne parvient pas toujours à dominer les événements. Sa patience, sa persévérance et son stoïcisme rythment le texte de Ferrari:

"Il n'a pas besoin de voir la mer pour rêver, les rêves de Marcel ne se nourrissent ni de contemplation ni de métaphore mais de combat, un combat incessant mené contre l'inertie des choses qui se ressemblent toutes, comme si, sous l'apparente diversité de leurs formes, elles étaient faites de la même substance lourde, visqueuse et malléable, (...) »(FERRARI, 2012: 67)

L'originalité du rêve de Marcel tient au fait qu'il ignore la médiocrité et la rêverie romantique. Par contre, il aime travailler et triompher non seulement de la maladie mais aussi de ce monde chaotique qui abonde en éléments dégoûtants et en "choses inertes". Il agit comme s'il était un surhomme porté aux changements géographiques ou géologiques. Si le paysage observé répugne à Marcel, c'est parce que ce dernier possède une volonté d'acier et qu'il ne se contente pas de percevoir les choses, mais il cherche plutôt à les transformer.

Par ailleurs, Marcel s'intéresse à ses études et refuse que son épuisement physique soit à l'origine de son échec intellectuel ou qu'il menace son avenir. $\mathrm{Au}$ contraire, l'infirmité dont souffre ce personnage ne le place jamais dans un rang dégradant mais elle devient un motif qui l'invite à travailler, à persévérer, à défier le destin et à surpasser les Autres en habileté:

"Il est le premier et le seul de ses frère et soeurs à poursuivre ses études au-delà du certificat et ni les démons de son corps ni l'inertie des choses ne l'empêcheront de les poursuivre jusqu'à l'École normale et encore au-delà, il ne veut pas être instituteur, il ne veut pas dispenser d'inutiles leçons à des enfants pauvres et sales dont le 
regard apeuré le renverra au désarroi de sa propre enfance (...) 》 (FERRARI, 2012: 68)

Bien qu'ils jouissent d'une bonne santé, le frère et les soeurs de Marcel n'ont pas pu continuer leurs études. Contrairement à ces comparses qui optent pour la facilité, Marcel se forge une carapace lui permettant de se protéger contre les aléas de la vie quotidienne. Il souhaite avoir une existence tout à fait originale, loin de la routine. Il aspire à toutes les métamorphoses salutaires aux plans moral, social, matériel, professionnel et psychologique. Il veut échapper à tout ce qui lui rappelle son enfance misérable. S'il refuse d'enseigner aux enfants, c'est parce qu'il ne veut pas rencontrer des clones ou des doubles destinés à renforcer chez lui le sentiment d'être maudit. Il souhaite aussi changer d'espace et s'installer à Marseille chez sa soeur aînée car son village natal acquiert un caractère dysphorique et carcéral (FERRARI, 2012: 72).

$\mathrm{Au}$ cours de son âge adulte, ce personnage se fie à l'idée sartrienne selon laquelle "l'homme est ce qu'il se fait":

"Il ne plaçait pas ses espoirs en Dieu mais dans les statuts de la fonction publique dont la bonne nouvelle venait d'être annoncée à tous les enfants de la République (...) » (FERRARI, 2012: 133)

D'après Marcel, il ne faut rien attendre de Dieu qui voue l'humanité à l'abandon et au néant. Il faut donc que l'être humain lutte pour se prouver et se réaliser. Mais malheureusement, Marcel ne récolte qu'un succès médiocre et relatif! Il n'aura jamais la gloire à laquelle il aspire.

Nous avons donc égard à une nouvelle philosophie: si nous, lecteur et chercheur, nous voyons que tout succès est quelque chose de positif et de bon, Marcel, lui, estime que la réussite sociale a des degrés. Selon lui, le succès qu'il obtient est insatisfaisant car le poste qu'on lui propose est celui d'un rédacteur dans les bureaux de l'administration dans une ville de l'Afrique. Ce métier exige que Marcel gouverne des africains barbares, un tel poste qu'il considère comme médiocre. En effet, ce personnage est- dès sa naissance- porté à l'émulation. Il souhaite se réfugier dans un passé glorieux et occuper une position à l'empire romain antique:

"(...) et il aurait mille fois préféré être le dixième ou le vingtième à Rome plutôt que de gouverner ainsi un royaume de désolation barbare des confins de l'Empire, mais personne ne lui offrirait jamais une telle alternative car Rome n'existait plus, elle avait été détruite depuis bien longtemps. » (FERRARI, 2012: 136)

Si le narrateur proscrit l'idée de vivre à Rome, c'est parce que Marcel est voué à un succès relatif. En d'autres termes, le succès absolu auquel il aspire n'existe pas. La médiocrité est le sort qui lui est réservé: 
" et celui qui fuyait sa misère ne pouvait rien espérer d'autre que d'exercer son pouvoir inutile sur des hommes plus misérables que lui, comme le faisait maintenant Marcel, (...). »(FERRARI, 2012: 136)

Ces quelques lignes vouent ainsi Marcel à un sort dépourvu d'éclat et de grandeur. Ne jouissant d'aucun prestige, il n'a qu'à imposer son pouvoir sur des créatures moins civilisées que lui. Contrairement aux héros du XXe siècle qui voient dans le voyage l'unique facteur de l'épanouissement, la fugue ne s'offre pas ici comme le moyen qui assure à l'être la promotion et la modification désirées. Au contraire, le voyage renforce chez lui le complexe d'infériorité. Même s'il possède un minimum de pouvoir, ce n'est qu'un « pouvoir inutile ». En d'autres termes, tout effort déployé ailleurs n'a aucune valeur; il ne mène qu'à un progrès limité destiné seulement à éloigner l'individu d'un passé

amer inhérent à son pays natal:

"Il travaillait à la préparation des concours en même temps qu'à se débarrasser des stigmates hideux de son passé, sa posture, sa démarche, son accent surtout, et il s'astreignait à rendre sa parole atone et limpide, comme s'il avait été dans le parc d'un manoir de Touraine, il affectait de prononcer son nom de famille en accentuant la dernière syllable (...). » (FERRARI, 2012: 133)

Le triomphe de Marcel n'est donc qu'extérieur et apparent. Il résulte d'un changement superficiel basé sur la modification de la manière de parler et de prononcer et non sur une véritable métamorphose morale et psychologique. Si cet homme veut se débarrasser des stigmates hideux de son passé (FERRARI, 2012: 133), c'est parce qu'il veut rompre avec un monde considéré comme caduc et hostile.

\section{La rupture avec un monde hostile.}

D'après Marcel, le monde où l'on vit est en proie à la déréliction:

"L'Archange avait depuis longtemps regagné son séjour céleste d'où il restait sourd aux prières et aux processions, il s'était détourné de ceux qui mouraient, à commencer par les plus faibles, les enfants, les vieillards, les femmes enceintes (...) » (FERRARI, 2012: 15)

Dès l'incipit du roman, le narrateur tend à démentir les paroles des prêtres qui glorifient le rôle de Dieu dans la vie humaine. S'il commence sa parole en citant des cas généraux qui souffrent de l'indifférence de la Providence, il finit sa méditation philosophique en faisant allusion à la réaction des parents de Marcel qui plaignent le sort de leur fils souffrant de l'ulcère:

"À chaque fièvre bénigne, à chaque nausée, à chaque quinte de toux, ils le veillaient comme un mourant, accueillant chaque guérison comme un miracle dont il ne fallait pas espérer qu'il se répète car rien ne s'épuise plus vite que l'improbable miséricorde 


\section{de Dieu. " (FERRARI, 2012: 16)}

Dès le début, le lecteur est donc plongé dans un climat métaphysique qui abonde en pessimisme et qui invite l'homme à ne pas avoir confiance en Dieu. La répétition du syntagme à chaque (FERRARI, 2012: 16) ne fait qu'accentuer la douleur physique de l'enfant Marcel ainsi que l'angoisse et la souffrance morale des parents qui attendent la mort prochaine et inévitable de leur fils, comme si Dieu trouvait son plaisir à voir les hommes souffrir et tourner à vide dans la spirale du tourment.

Pour l'adulte Marcel, la maladie devient un motif de craindre les Autres et l'infirmité physique, dont il est l'objet, se transforme en une obsession hypocondriaque qui le pousse à ressasser ses peines à chaque fois qu'il regarde son image dans le miroir:

"(...) et quand il contemple sa propre silhouette dans le miroir, Marcel a le sentiment qu'il est né ainsi, vacillant et maigre, et que l'enfance l'a marqué d'un sceau cruel dont rien ne pourra le libérer. »(FERRARI, 2012: 68)

Le miroir se transforme alors en un alter ego qui livre une réalité pure à l'être, une réalité dénuée de toutes les fioritures ainsi que de toutes les apparences trompeuses. Grâce à ce miroir, Marcel parvient à se juger, à se connaitre et à ressentir l'impact de son affaiblissement physique sur son temparément et sa psychologie.

En contemplant sa silhouette au miroir, Marcel ne nous rappelle-t-il pas Narcisse qui se complaisait à voir son double dans l'eau? Mais à la différence de Narcisse qui se remplissait d'orgueil, Marcel voit s'accentuer chez lui le sentiment d'infériorité car il devient l'esclave d'une grave maladie susceptible de l'affaiblir et d'entraîner une altération de ses rapports humains avec l'entourage car une personne chétive ne jouit pas de la même force ou $\mathrm{du}$ même charisme qu'une personne valide et robuste. Contrairement à Narcisse qui- par sa maladresse- a provoqué sa propre noyade en voulant tuer son double, Marcel est très conscient que le double apparaissant dans le miroir n'est que lui-même. Marcel connaît bien sa problématique et il sait bien apprécier ses compétences. En un mot, il sait ce qu'il veut (accomplir un grand succès) mais il ne peut pas réaliser son but en raison de la malédiction initale qui pèse sur son destin dès son bas âge et à cause de l'incompatibilité éternelle qui existe entre le monde et son épanouissement moral:

"(...) car le monde persistait à contrarier ses rêves au moment même où ils devenaient réels. " (FERRARI, 2012: 133)

La maladie dont souffre cet homme devient le véritable opposant qui le prive de la tranquillité d'âme et de la sérénité morale et qui l'invite à être phobique et craintif:

"(...) Marcel scrutait ses vomissements, ses urines, ses selles, avec

la peur panique d'y découvrir des grappes dorées de larves, 
d'araignées, de crabes ou de couleuvres, et il attendait de mourir seul, transformé en pourriture avant même de mourir. " (FERRARI, 2012:141)

Les lignes ci-dessus suscitent chez le lecteur des sensations désagréables provenant de l'énumération des sécrétions et des excréments humains. Nous avons donc égard à une image prosaïque qui abonde en éléments quotidiens et banals, qui se double aussi de quelques détails monstrueux. En effet, les insectes ou les animaux dont rêve Marcel témoignent de ses tourments et de ses craintes superstitieuses. Il est donc temps d'être réaliste, d'apprécier ses propres aptitudes physiques et corporelles et de renoncer à sa naïveté et à son ignorance d'autrefois:

"(...) c'en est fini des luttes épiques, il faut se contenter d'ennemis méprisables, la grippe, les rhumatismes, la dégénérescence, les courants d'air dans le grand appartement du 8e arrondissement (...). " (FERRARI, 2012: 144)

Nous avons donc égard à un personnage qui reconnaît son erreur et qui déclare solennellement sa défaite et son échec face au destin inexorable. Si Ferrari a commencé son roman par souligner le stoïcisme de Marcel en supportant la maladie et la douleur, la seconde moitié du Sermon sur la chute de Rome a été consacrée au dénigrement de cet être impliqué dans un " roman imbu de solitude et d'impuissance " (GLAUDES \& al.,1991: 114). Les critiques littéraires vont même jusqu'à faire allusion à cette dualité qui caractérise tous les personnages romanesques:

«Dans leur solitude ces personnages à la recherche d'eux- mêmes apprennent que ce moi qu'ils exaltent est un abîme de contradictions, une entité insaisissable (...). » (GLAUDES \& al., 1991: 86).

Le passage ci - dessous dévoile davantage les postulations contradictoires entre lesquelles cet anti-héros est tiraillé:

"Il tenait le journal intime de sa maladie dont il notait scrupuleusement tous les symptômes, (...), il pensait à sa jeune épouse, à ses cuisses autour de son visage, et elle lui semblait alors si vivante qu'il la désirait avec passion, et il notait alors, délire, priapisme, nécrophilie, nostalgie létale (...). » (FERRARI, 2012:141)

Marcel éprouve donc des sentiments antithétiques allant de l'extase au désenchantement, de la souffrance à la quête du plaisir. Le lecteur éprouve, à son tour, une confusion à l'égard de cet anti-héros qui semble tâtonner dans les ténèbres et qui devient inapte à déterminer ses priorités. On se pose la question suivante: Pourquoi Marcel n'a-t-il pas sollicité l'aide d'autrui pour dépasser sa crise? En effet, jusqu'à maintenant, nous sommes emprisonnés dans la conscience interne d'un seul individu. Nous ignorons si les Autres 
peuvent jouer un rôle thérapeutique et positif à son égard ou bien s'ils accentuent son malaise.

Si nous sommes enclin à voir qu'il y a un conflit entre Marcel et la société, c'est parce que les Autres sont les bourreaux de l'individu. Nous tendons ainsi à reprendre la célèbre expression sartrienne "L'enfer, c'est les Autres" mais aussi à appuyer la théorie de Michel Zéraffa qui dit:

"Le roman du XXème siècle présente le plus souvent des héros ayant à cour non pas d'échapper au monde (ils en sont les victimes consternantes) mais de s'y soustraire (...). Dans tout le roman moderne, rapports sociaux et rapports humains sont montrés comme incompatibles. 》 (ZÉRAFFA, 1971: 70)

Toute comparaison faite entre Marcel et les Autres n'est pas en sa faveur. En effet, ce personnage éprouve un tel sentiment d'infériorité à l'égard d'André (son beau-frère) qu'il a honte de l'accueillir dans son village natal (FERRARI, 2012: 83). En outre, le succès de ce beau-frère accentue chez Marcel les sentiments de médiocrité et d'infériorité:

" (...) il a honte devant André d'avoir été un si piètre guerrier, et il a honte de sa chance méprisable et encore honte de n'être pas même capable de s'en réjouir, il regarde André avec un respect envieux. » (FERRARI, 2012: 83)

À l'échec social et professionnel de Marcel s'ajoute le regard bourreau d'autrui qui le juge et le condamne pour sa défaite et son impuissance. Si Marcel est un piètre guerrier, c'est parce qu'il a été obligé - au début de sa carrière militaire- d'être un scout occupé à chanter la gloire du Maréchal (FERRARI, 2012: 72), comme si les officiers ne pouvaient pas compter sur lui pour accomplir un travail militaire digne d'importance, comme si Marcel était condamné à chanter et à louer les exploits d'autrui sans avoir le droit d'y prendre part.

Ce qui empire la situation de ce personnage aux yeux de ses supérieurs, c'est qu'il accepte d'être un officier d'intendance au lieu d'être un officier d'artillerie. Il n'est donc pas prêt à mourir en faveur d'un idéal. Ce qui compte pour lui, c'est de survivre même si cela est aux dépens de la morale et de l'honneur. Le dialogue ci-dessous qui se déroule entre Marcel et son colonel fait de celui-là une marionnette qui ne songe point aux intérêts publics mais uniquement aux siens:

"Il se sentait enfin heureux et il le demeura jusqu'à ce que le colonel le convoquât pour lui reprocher son ignominie en des termes d'une violence inouïe, il écumait, il tapait du poing sur son bureau, vous n'êtes qu'un petit fumier, Antonetti, doublé d'un lâche, et Marcel, éperdu, bafouillait en vain, mais mon colonel, mon colonel et le colonel hurlait, officier d'intendance? d'intendance? répétant le mot" intendance" comme s'il s'agissait d'une obscénité sans nom qui lui 
souillait la bouche, vous avez peur de vous battre, c'est ça? 》

(FERRARI, 2012:80-81)

Il ne faut pas omettre ici l'emploi du langage familier et injurieux dans le syntagme "petitfumier 》 car ce terme reflète le dépit du colonel et passe pour une arme qui réduit au néant toute tentative de la part de Marcel pour se défendre ; la preuve en est que celui-ci n'a trouvé aucun mot pour se disculper: " il bafouillait en vain ».

À la sous-estimation de Marcel par ses collègues, s'ajoute le mépris des membres de la famille à son égard. Tout le long du roman, Marcel passe son temps à contempler ses proches qui existent dans la photo de l'absence ${ }^{(1):}$

"Il les toise parfois avec colère, il a envie de leur reprocher leur manque de clairvoyance, leur ingratitude, leur indifférence, (...) 》 (FERRARI, 2012:13)

En continuant la lecture de la page, nous serons surpris par la note du narrateur qui déclare que Marcel ne manqua à personne et qu'aucun membre présent dans cette photo ne pensa jamais à lui (FERRARI, 2012:13). Si le narrateur n'énumère ni les causes ni les manifestations de cette ingratitude et de cette indifférence, c'est parce qu'il invite le lecteur à appliquer sa raison et à déployer un effort dans le décryptage de ce message codé. Peut-être ces proches ont-ils agi de cette manière égoïste parce que Marcel est malade et qu'il est frappé dès son bas âge par un ulcère car «la différence physique, en effet, est en général mal accueillie.» (BERTHELOT, 1997: 92). Plutôt que de contenir le malade, de le protéger et de l'entourer de soins, ces proches l'évincent, le repoussent et l'excluent de leur sphère sociale, comme s'ils avaient honte d'avoir dans leur famille un malade et comme si la maladie était un facteur qui ne leur apporte qu'opprobre et humiliation. En effet, Marcel vit dans un milieu familial où est proscrit tout sentiment euphorisant, où " l'amour lui-même est absent. » (FERRARI, 2012:19).

Marcel s'habitue donc à voir ses proches d'un oeil tout à fait différent et à les juger de façon subjective destinée à refléter sa colère et son indignation. S'il déteste son frère Jean-Baptiste, c'est parce que celui-ci jouit de la vitalité alors que lui, Marcel, manque de virilité. Il se prend pour un être faible et médiocre; le succès de Jean-Baptiste ne sert donc qu'à amplifier l'échec de Marcel.Cet anti-héros va jusqu'à envier son frère

(1) La photo de l'absence est une photo prise par un photographe ambulant pendant une journée caniculaire de l'été 1918. Elle est surnommée la « photo de l'absence » car tous les membres de la famille y sont présents sauf Marcel qui était dans le ventre de sa mère.

qui jouit du respect des Autres ainsi que de la protection divine dont lui, Marcel, a été privé: 
"Marcel ne l'admirait plus, il le jalousait si ostensiblement d'un trésor qu'il ne méritait pas. Tout ce qu'il voyait de son frère lui était devenu insupportable, son goût manifeste pour les putains, sa carrure imposante, sa maigreur et sa graisse, l'insolence de son attitude, sa générosité même, car tout cet argent ne pouvait pas avoir été économisé sur sa solde de sergent - chef et devait sans aucun doute provenir de trafics abominables, de piastres, d'opium ou de chair humaine. " (FERRARI, 2012:69)

C'est ainsi que cet anti-héros est obligé de ressasser ses tourments et ses rancoeurs à l'égard de son frère qui jouit de la gloire et de la santé, alors que lui, Marcel, sombre dans la maladie. Quant aux succès de Jean-Baptiste, ils sont mal interprétés par Marcel qui éprouve une méfiance considérable à l'égard de ce frère. Selon lui, toute métamorphose salutaire subie par JeanBaptiste s'explique par l'escroquerie de ce dernier, ce transgresseur des lois, cet être parasitaire qui aspire à la richesse, abstraction faite de la morale.

Même le mariage de Marcel n'a pas pu mettre un terme à ses envies et à ses tourments. Si Jean-Baptiste renforce chez cet anti-héros le complexe d'infériorité, la femme de Marcel provoque aussi sa honte en public:

" et Marcel aurait été parfaitement heureux si l'inquiétude ne l'avait pas submergé à chaque fois qu'il devait paraître à la table du bord avec sa femme, il avait constamment peur que quelqu'un lui posât une question anodine à laquelle elle répondrait si bêtement que toute la tablée en demeurerait silencieuse à moins qu'elle ne répondît pas du tout et ouvrit une bouche toute ronde de surprise avant de baisser les yeux en gloussant (...). " (FERRARI, 2012: 132)

Marcel estime donc que cette femme est indigne de lui et qu'elle constitue un obstacle important qui pourrait bien entraver son ascension sociale. Plutôt que de l'entourer de soins, de lui accorder le courage et de l'inviter à continuer son succès, cette épouse est perçue comme un opposant qui compromet l'avenir de son mari. Elle est toujours sous-estimée par Marcel qui voit en elle une gangrène apte à menacer sa sécurité professionnelle et sa position sociale:

" et il était au supplice à chaque fois qu'elle lui parlait en public, il avait honte qu'elle s'adressât à lui en corse, ce patois ridicule dont il n'arrivait pas à chasser les sonorités insupportables, et il était en même temps soulagé parce que personne ne pouvait comprendre ce qu'elle disait et il attendait le moment où il pourrait refermer la porte de leur cabine sur une intimité qui seule venait à bout de ses rancoeurs et de ses tourments. " (FERRARI, 2012: 132)

Même la parole et la voix de cette femme lui répugnent. Le langage qu'elle emploie provoque chez lui des sensations auditives désagréables. La présence de cette personne (qui est censée être une consolatrice) ne fait qu'accentuer le 
tourment de son mari et accélérer sa chute et son déclin. À chaque comportement qu'elle adopte, à chaque commentaire qu'elle fait et à chaque mot qu'elle prononce, Marcel considère que sa réputation et son image seront ternies aux yeux des Autres. Toutefois, il reçoit un choc considérable lorsqu'elle est morte en couches (FERRARI, 2012: 31). Mais à cette mort s'ajoutent d'autres décès. C'est ainsi que le texte de Ferrari est rythmé par la mort.

Tout le long du roman, Marcel ne fait qu'enterrer les proches qui meurent: "Le temps s'est allégé de l'espoir et il file, imperceptible et vide, au rythme toujours plus rapide des enterrements qui rappellent Marcel au village, comme si sa seule mission constante en ce monde était de porter les siens en terre, les uns après les autres, sa femme repose maintenant en Corse, (...), et meurent ses soeurs aînées, les unes après les autres, dans l'ordre exact fixé par la sagesse de l'état civil, à Paris, la saveur de la solitude se fait peu à peu insipide (...). » (FERRARI, 2012: 144)

Le thème classique de la fuite du temps obsède alors Ferrari qui met en scène un personnage choqué par la loi de l'absurde et du néant gouvernant le monde. Personne ne pourra échapper au pouvoir inéluctable du temps. Tout le monde connaîtra tôt ou tard la mort prochaine et inévitable ainsi que l'oubli définitif. Dans la scène-ci dessous, nous assistons à la mort des parents de Marcel:

"Marcel rentra au village pour enterrer son père, puis sa mère.

Et il ne les pleura pas parce que la mort avait toujours été leur vocation et il était presque heureux qu'ils aient enfin pu répondre à un appel qu'ils avaient dî feindre si longtemps de ne pas entendre. » (FERRARI, 2012: 143)

Cet exemple fait des parents de Marcel des êtres prédestinés à la mort. La lutte épique contre la mort n'a pas réussi à prolonger leur existence puisqu'ils ont dû enfin se soumettre à une loi imposée par la fatalité invincible. Si Marcel ne pleure pas ses parents, c'est parce que le deuil et le regret ne servent à rien. Les larmes versées changeront-elles la réalité? Il s'agit plutôt d'être réaliste et de reconnaître son impuissance face au destin. Pourquoi donc Marcel pleurera-t-il les morts alors que le passage ci-dessous affirme qu'il est mortel, lui aussi?:

"À présent qu'il a porté les siens en terre, l'un après l'autre, la mission harassante qu'il a accomplie doit échoir à un autre, et il attend que sa santé toujours chancelante et inaltérable soit finalement vaincue car, dans l'ordre fixé par l'état civil, son tour est maintenant venu de marcher seul au tombeau. " (FERRARI, 2012:146)

Devenu un vieillard décrépit, Marcel ne meurt pas dans le roman mais sa mort inévitable et certaine est relatée grâce à des signes prémonitoires. Le 
passage ci-dessus fait de cette mort le produit logique de tous les événements relatés au sein du livre. À l'ulcère gastralgique qui tourmentait Marcel depuis son enfance s'ajoute le vieillissement actuel.

Alors que la fin prémonitoire de Marcel s'avère logique à nos yeux, la mort de Jacques (son fils) est considérée comme choquante, scandaleuse et démoralisante car il s'agit d'un fils qui est mort avant son père:

" il était dans l'ordre des choses que les enfants enterrent leurs parents mais (nous soulignons) le bouleversement intolérable de cet ordre ajoutait le scandale à la douleur. " (FERRARI, 2012:167)

Le connecteur " mais " fait de cet ordre des choses un leurre, voire un désordre qui gouverne ce monde aléatoire et chaotique où " il ne faut jurer de rien " (selon les termes de Musset) et où l'on ne peut rien prévoir. Tout est donc soumis aux caprices de la fatalité. Tout est précaire. La seule chose stable dans cet univers est la banalité, renforcée par le phénomène de l'éternel retour:

"Mais rien ne se passait, un monde avait bel et bien disparu sans qu'aucun monde nouveau ne vienne le remplacer (...). » (FERRARI, 2012: 17)

En effet, il n'y a rien de nouveau dans ce monde. Tout se répète de façon mécanique. Aucun changement radical ne caractérise l'existence humaine. Aucune innovation morale et psycholgique n'est perçue comme un exploit proprement dit. Selon Mircea Eliade, ce que l'être considère aujourd'hui comme un nouveau phénomène n'est, en effet, que la répétition ou l'imitation d'un ancien événement:

"Le passé n'est que la préfiguration du futur. Aucun événement n'est irréversible et aucune transformation n'est définitive. Dans un certain sens, on peut même dire qu'il ne se produit rien de neuf dans le monde, car tout n'est que la répétition des mêmes archétypes primordiaux» (ELIADE, 1969: 107)

Même le monde actuel n'est qu'une répétition déformée et indirecte du monde archaïque:

"(...) rien ne se passait, le temps n'apportait rien de plus que la succession monotone de saisons qui se ressemblaient toutes et ne promettaient que la malédiction de leur permanence, (...) tous les miroirs reflétaient des regards semblables, les mêmes abîmes creusés dans des visages de cire. " (FERRARI, 2012: 17)

Cet éternel retour empoisonne donc à l'être son existence et lui ôte toute signification en soi. Toute répétition étouffe la créativité humaine et déforme les identités car elle rend tous les êtres identiques mais aussi inertes et indifférents face au progrès ou à la chute, face à la vie ou à la mort. À quoi bon se distinguer et se forger un destin exceptionnel puisque chaque individu n'est que le sosie de son semblable? Les deux naissent, vivent et meurent. 
Chacun ne fait que continuer "la comédie de la génération et de la mort » (FERRARI, 2012: 17).

Au-delà de la dimension cosmique de l'éternel retour, ce phénomène dispose d'un arrière-plan littéraire ou culturel. Il laisse une profonde empreinte sur Jérôme Ferrari qui éternise l'archétype de l'anti-héros dans Le sermon sur la chute de Rome. Même si Marcel va mourir, il continuera à exister dans le texte littéraire, et ce grâce à ses doubles car toutes les vies particulières, au-delà de leurs différences, sont structurellement, analogues (JOUVE, 2001: 135).

\section{L'éternel retour de l'anti-héros.}

Dans le roman de Ferrari, l'anti-héros est un portrait récurrent et multipliable. L'altération du moi passe pour une malédiction héréditaire qui se transmet du grand - père (Marcel) à son petit - fils (Matthieu). Si le récit de l'échec de Marcel finit mal, son histoire ne finira point:

"Tout nimbés du pessimisme schopenhauerien, les divers romanciers vont proposer des récits de l'échec, du ratage, de l'impuissance. Les histoires qu'ils imaginent et attribuent à leurs héros sont de celles qui, tout à la fois, finissent mal et ne finissent pas, dans une sorte de double avortement. " (GLAUDES \& al.,1991: 109).

Cet archétype universel ne mourra donc jamais. Il demeurera éternel et fournira à la littérature toute une matière fertile.

Tout d'abord, Matthieu semble coupé du monde. Sa qualité en tant que membre dans une communauté est mise en cause puisqu'il souffre de la solitude:

"Matthieu avait dix-huit ans quand sa mère, inquiète de son caractère résolument solitaire et méditatif, décida qu'il lui fallait un ami pour profiter de ses vacances au village. " (FERRARI, 2012:29)

C'est la mère qui modèle alors la personnalité de son fils sur son propre goût. C'est elle qui lui choisit les amis car elle le trouve incapable de faire des choix fructueux et réussis. L'indication portant sur l'âge de Matthieu (qui a dix-huit ans) fait de lui un enfant éternel, un homme dépourvu de toute virilité. Il dépend toujours de sa mère qui exige une métamorphose de son comportement peu grégaire. Incapable de faire un examen de conscience ou de se rendre compte de ses défauts, il laisse les Autres intervenir dans sa vie:

"(...) on l'avait condamné à vivre une vie d'étranger, sans même qu'il pût s'en rendre compte, une vie dans laquelle tout ce qui lui était familier était devenu haïssable et qui n'était même pas une vie, mais une parodie mécanique de la vie, qu'il voulait oublier, en laissant par 
exemple le vent froid de la montagne fouetter son visage (...). » (FERRARI, 2012:35)

Plutôt que d'agir, Matthieu ne fait que subir. N'ayant aucune prise concrète sur le monde, il souffre de l'aliénation ainsi que de la perte d'identité. La seule solution qui lui permet d'échapper à cette aliénation réside dans une amnésie susceptible de lui faire oublier son existence insupportable; idée suggérée par la dernière phrase du passage ci-dessus. Même en pensant à la métamorphose, il ne songe qu'aux actions négatives, voire à l'inaction car à nos yeux, l'amnésie n'est qu'une passivité lui permettant de répudier le passé, de s'amputer d'une partie de soi et de se réfugier dans l'oubli. Même le procédé qui l'aidera à subir cette amnésie relève d'une solution puérile " $i l$ voulait oublier [sa vie], en laissant par exemple le vent froid de la montagne fouetter son visage " (FERRARI, 2012: 35). Matthieu a donc un objectif médiocre qu'il souhaite réaliser par le biais d'un moyen banal et illusoire. En effet, est-ce que le vent froid qui fouette le visage permettrait-il à l'être d'oublier son passé?

Nous avons donc égard à un anti-héros qui ne se fie pas à ses aptitudes physiques ou intellectuelles pour réaliser un véritable changement dans sa vie. S'il souffre d'une carence intellectuelle, c'est parce qu'il a interrompu ses études universitaires qui, à ses yeux, ne déboucheraient sur rien. Certes, ce manque d'instruction a fait de lui un être dépourvu d'éclat et d'intelligence, un homme à l'esprit étriqué qui se fie uniquement au sens concret des termes et à l'apparence trompeuse des objets:

"Peut-être aurait-il pu pleurer s'il avait compris le langage des symboles, ou s'il avait au moins pu faire un effort d'imagination mais il ne comprenait rien, et il n'avait plus d'imagination, son esprit butait sur la présence concrète des choses qui l'entouraient, au-delà desquelles il n'y avait rien. » (FERRARI, 2012:154)

Matthieu n'est donc qu'un enfant inapte à la compréhension, à la justification, à l'explication, à l'interprétation ou même à la communication. L'ignorance lui permet de vivre à l'aveuglette et de survivre à la douleur morale tout en ne s'inquiétant de rien (FERRARI, 2012: 90). En effet, son indifférence ne fait de lui qu'une bête (FERRARI, 2012:154).

Même en travaillant et en cherchant à gérer un café en Corse, Matthieu s'avère passif puisque son choix ne relève pas d'une attitude sincère et réfléchie (FERRARI, 2012: 89). Incapable de déterminer ce qu'il veut, il se livre à une métamorphose sur tous les plans, mais il demeure incapable de calculer les conséquences de ses actes. S'il se lance dans ce projet, c'est pour échapper à la vie qu'il menait auprès de ses parents, à cette existence qui se caractérisait par la médiocrité:

"Matthieu se comportait comme s'il lui fallait s'amputer de son passé, il parlait avec un accent forcé qui n'avait jamais été le sien, un 
accent d'autant plus ridicule qu'il lui arrivait de le perdre au détour d'une phrase avant de se raviser en rougissant et de reprendre le cours de sa grotesque dramaturgie identitaire d'où la moindre pensée, la plus petite manifestation de l'esprit étaient exclues comme des éléments dangereux » (FERRARI, 2012: 89)

Cet anti-héros qui tâtonne alors dans les ténèbres ne maîtrise pas l'art de parler. Quant à la modification de son accent, elle témoigne uniquement d'un changement extérieur de sa nature. Pourtant, l'intérieur demeurera à jamais fade et stérile car Matthieu se fie à la philosophie de l'éternel retour:

"(...) il attendait que la nuit tombe et que la vie reprenne, sans nouvelle surprise, car il était redevenu un petit enfant qui ne se rassure que dans la perpétuelle répétition du même (...). " (FERRARI, 2012:162)

C'est dans le vide que Matthieu retrouve donc sa plénitude. En d'autres termes, le déclin fait son épanouissement moral. La routine quotidienne l'aide à avoir plus de confiance en l'avenir, et ce en lui donnant l'impression que tout ce qui lui arrivera de bon ou de mauvais n'est qu'une répétition d'un ancien phénomène remontant au passé. Pourquoi donc Matthieu s'intéresserat-il à son avenir? En fait, nous avons égard à une philosophie qui met tous les hommes sur le même pied d'égalité, qui rend vaine et stérile toute innovation et qui provoque une amertume dans l'esprit humain. Cependant, c'est cette philosophie pessimiste qui fait le bonheur de Matthieu vu qu'elle le débarrasse de tous les soucis qui pourraient l'assaillir.

Sombrant dans la médiocrité affichée par cette philosophie, cet anti-héros pense bien que sa présence au chevet de son père (Jacques) ne ralentira point la mort de celui-ci ni ne prolongera son existence. Aussi Matthieu ne visite-til pas Jacques au cours de sa maladie létale.

Si le portrait de Jacques demeure caché au lecteur, sa présence sert à dévoiler l'opportunisme et l'irresponsabilité de Marcel (son père) et Matthieu (son fils). Il passe toujours pour un fardeau dont tous les deux cherchent à se débarrasser à tout prix. Au cours de l'enfance de Jacques, Marcel l'a abandonné et l'a confié à sa soeur Jeanne-Marie pour prendre soin de lui (FERRARI, 2012: 31). Pendant sa vieillesse et au cours de sa maladie (l'insuffisance rénale dont il souffrait), Matthieu n'a pas pensé à aller le voir à l'hôpital. Il n'est venu qu'au cours de l'enterrement, comme si Jacques n'était pas son père (FERRARI, 2012: 120).

Tous deux, Marcel et Matthieu, éprouvent alors le même mépris à l'égard de ce personnage. Tous deux refusent de déployer le moindre effort pour sauver la vie à cette victime. Au contraire, ils s'efforcent de lui empoisonner l'existence et d'accentuer sa souffrance et sa solitude. Plutôt que de veiller sur Jacques, de le consoler et de le protéger, Marcel et Matthieu renforcent chez lui le sentiment d'être mal aimé. En effet, comment protégeront-ils les 
Autres alors qu'eux-mêmes, ils sont faibles et vulnérables et qu'ils ont besoin d'être protégés, non contre un danger extérieur, mais contre une faiblesse intérieure, faite de paresse et d'impuissance communes?

Conformément aux théories de la narratologie classique, ces deux personnages sont considérés comme des "antagonistes 》 (BERTHELOT, 1997: 126) qui refusent d'assister le faible ou le vulnérable pour qu'il puisse se remettre de sa crise. Ils ignorent les simples valeurs humaines, n'apprécient guère le sens de la solidarité ou de l'action charitable et n'ont aucun sentiment de sympathie à l'égard de

leurs proches.

Certes, l'indifférence de Matthieu à l'égard de son père a provoqué la colère et l'indignation de sa soeur Aurélie qui l'avait critiqué de façon acerbe en lui disant:

" qu'il n'était qu'un petit con répugnant d'égoïsme, un petit con aveugle qui espérait au fond de lui que son aveuglement finirait par lui valoir l'absolution, mais jamais il ne serait absous de ce qu'il était en train de faire. " (FERRARI, 2012: 117)

D'ailleurs, elle voyait en lui "un être sans envergue ni intérêt " (FERRARI, 2012: 89), voire " un enfant gâté, un petit morveux 》 (FERRARI, 2012: 88).

Ces épithètes péjoratives ont eu un écho sur Matthieu qui essayait, tout le long du roman, de trouver une issue à sa crise à travers quelques simples moyens. Tout d'abord, il s'imagine qu'il est "un héros

redoutable » (FERRARI, 2012: 39) en écoutant Virgile (un de ses amis) dire que son père était l'adversaire redoutable des Italiens au cours de la seconde guerre. Les ambitions de ce jeune homme relèvent donc de l'onirisme car ce type de personnage ne fait que rêver d'une condition meilleure que celle dont il jouit. Il ne cherche qu'à envier les Autres et à percevoir leur ascension, sans avoir l'audace de les imiter ni de prendre l'initiative de combattre un ennemi. Les désirs et les rêves héroïques de ce personnage ne se transforment jamais en actes concrets. En effet, cet anti-héros ne peut accomplir aucune action positive ou remarquable car toutes ses actions sont puériles, comme en témoigne la scène ci-dessous:

"Aurélie avait presque de la peine pour son frère, il avait vraiment l'air d'un enfant et il n'était au fond qu'un enfant, exaspérant et vulnérable, qui ne pouvait se protéger de la menace des cauchemars qu'en se réfugiant dans un monde irréel des rêves puérils, un monde de sucreries et de héros invincibles. " (FERRARI, 2012:168)

Le jugement qu'on émet sur cet anti-héros ne reçoit sa valeur que de la polyphonie qui contamine le passage ci-dessus. En effet, le fait d'évaluer le comportement médiocre de Matthieu à travers un discours indirect libre ne fait qu'accentuer la vigueur du drame et doubler la problématique du 
personnage. Si Matthieu est lâche aux yeux de sa soeur, il l'est également aux yeux du narrateur. Ce dernier s'infiltre dans le subconscient de l'anti-héros et essaie d'analyser sa psychologie et son état d'âme. Il finit par conclure que Matthieu échappe à la médiocrité de son existence en recourant aux activités ludiques ou aux solutions néfastes et grossières qui aggravent l'ampleur du drame au lieu de l'éradiquer. L'exemple ci-dessous met l'accent sur le rôle de l'alcool dans la vie de Matthieu:

"Matthieu n'avait rien d'autre à faire qu'à savourer son bonheur en buvant l'alcool qui lui brûlait les veines. De temps en temps, quand elle avait décidé que c'était son tour, il couchait avec Virginie Susini (la fille de la propriétaire du café). » (FERRARI, 2012: 100)

L'alcoolisme et le sexe deviennent donc les seules échappatoires permettant au personnage de vaincre son destin. Son caractère solitaire ne l'empêche pas de nouer des rapports avec les serveuses du café. En effet, si l'anti-héros décide de s'ouvrir aux femmes, c'est parce qu'il cherche uniquement à satisfaire ses désirs sexuels:

"Le personnage décadent choisit de vivre dans un isolement où se complaît son narcissisme. Il réduit son besoin des autres à très peu. Sa seule perplexité a trait au partenaire amoureux ou sexuel.
Il ne peut faire fi du désir (...). »
(GLAUDES \& al., 1991: 110)

C'est ainsi que Matthieu se livre uniquement à la débauche et qu'il se voue à une quête effrénée du plaisir physique, comme en témoigne l'exemple ci-dessous:

"Quand il se réveilla, sa tête reposant contre les seins d'Isazkun et l'une de ses mains était posée sur les hanches d'Agnès. Il prit un café et rentra chez lui pour se doucher. Mais il n'y dormit plus jamais. Le lendemain, il coucha avec Rym et Sarah. " (FERRARI, 2012: 103)

Ce n'est donc pas une seule femme qui l'invite au rapport sexuel, mais elles sont quatre jeunes filles. Ce qui compte pour cet homme lubrique et inconstant, ce n'est pas l'amour au sens exact, mais seulement le plaisir physique découlant du rapport charnel. Incapable de trouver une femme qui puisse le comprendre, l'entourer de soins et le contenir, il décide de connaître plusieurs femmes à la fois. À ses yeux, chacune a sa méthode dans l'amour, ce qui lui permet de connaître un plaisir intense, un plaisir procuré grâce à des femmes originales qui lui épargneraient l'amour fade et routinier qui pourrait être donné par une seule femme. En effet, dès le début, ce personnage a déclaré qu'il était incapable de se marier ou de fonder une famille:

"(...) il était incapable de nouer une relation amoureuse avec qui que ce soit, du moins ici, à Paris, parce qu'il n'était pas destiné à y demeurer et ne voulait mentir à personne. Et c'est ainsi qu'au nom 
d'un avenir aussi inconsistant que la brume, il se privait de présent, comme il arrive, si souvent, il est vrai, avec les hommes. " (FERRARI, 2012: 49-50)

En faisant donc l'amour avec plusieurs femmes, Matthieu prouve que le célibat n'est pas un obstacle susceptible d'entraver sa quête hédoniste. Au contraire, cette condition n'est qu'un catalyseur ou un adjuvant qui favorise sa recherche de la concupiscence. Il peut même voir dans le célibat une occasion pour avoir plusieurs maîtresses et pour éprouver une jouissance physique à plusieurs reprises car il paraît que les sirènes, avec qui il couche, maîtrisent bien l'art de la séduction.

Au-delà de cette tendance à la débauche, Matthieu s'est transformé en un être violent. Après avoir pris la gérance du café, il a dû se défendre contre le danger, contre les voleurs qui pourraient l'attaquer surtout que le café était situé dans une région reculée. C'est pourquoi il était armé d'un pistolet, et le dialogue ci-dessous qui se déroule entre Matthieu et sa soeur révèle les tendances sanguinaires de cet anti-héros:

" Aurélie le considéra avec stupeur.

Tu as un pistolet maintenant? Tu deviens complètement dingue? Tu as quoi? Des problèmes de virilité? Et c'est ridicule, par-dessus le marché. Tu t'en rends compte? 》(FERRARI, 2012: 106)

Ces multiples interrogations révèlent le choc d'Aurélie qui ne comprend pas la raison pour laquelle son frère cherche à devenir un criminel, un assassin. Elle a l'impression qu'il lui est totalement étranger. Si Aurélie voit que son frère a " des problèmes de virilité », c'est parce qu'elle est dotée d'un pouvoir qui lui permet de comprendre la nature de ce personnage décadent, lequel cherche à prouver sa virilité par l'agressivité et la témérité. Si, au début de notre étude de ce personnage, nous avons mis l'accent sur ses attitudes puériles dans la solution de ses problèmes, nous révélons maintenant ses tendances belliqueuses, comme si la raison et le bon sens ne caractérisaient point les choix ou les actes de cet anti-héros, comme s'il était condamné à jamais à l'ignorance qui l'oblige à préférer l'ombre à la lumière, le profane au sacré, l'avilissement à la sagesse, l'illusion à la réalité.

Si Matthieu recourt à la violence pour se protéger, c'est parce que " l'homme est un loup pour l'homme" (selon les termes de Thomas Hobbes). Personne n'aime personne. Personne ne s'intéresse à personne. Autrui qui est considéré comme mon "prochain" ou mon "semblable" n'est, à vrai dire, que mon pire ennemi cherchant à me supprimer, à m'évincer ou à me tuer car le faible n'a pas de véritable place dans cette société régie par les lois de la jungle. Une dernière situation tirée du roman suffit de trancher la situation et de révéler la sauvagerie des hommes qui recourent au machiavélisme et qui font fi du bon sens: 
"À nouveau, le monde était vaincu par les ténèbres et il n'en resterait rien, pas un seul vestige. À nouveau, la voix du sang montait vers Dieu depuis, dans la jubilation des os brisés, car nul homme n'est le gardien de son frère, et le silence fut bientôt suffisant pour qu'on pût entendre le hululement mélancolique de la chouette dans la nuit d'été. » (FERRARI, 2012: 191)

Dans ce passage, il s'agit d'une méditation de la part de Matthieu à la suite d'un meurtre commis par Libero (son ami et son partenaire dans la gérance du café) qui a commis un meurtre afin de mettre un terme à une dispute entre Pierre-Emmanuel et Virgile (deux employés dans le café), et ce en tuant ce dernier. Au-delà de la laideur de l'image ci-dessus, il y a une sorte d'humour noir qui découle de ce meurtre: nous avons égard à un homme (Libero) qui recourt à un acte barbare pour conserver l'ordre dans le café. Afin de créer une amibance de paix et de sécurité, faut-il donc recourir au pistolet et à la violence? Faut- il faire couler le

sang ou tuer quelqu'un pour mettre un terme à une querelle?

Il paraît donc que le débat et le dialogue sont méconnus par Libero qui passe lui aussi pour un troisième anti-héros. Ce personnage s'est lié d'une profonde amitié avec Matthieu et a été en proie à la même malédiction ayant fait de lui un être médiocre, prêt à tout pour rejoindre son ami:

"Et Libero, (...), semblait résolu à suivre le même chemin, se contentant de répondre par une onomatopée vaguement interrogative quand elle (Aurélie, soeur de Matthieu) lui apprit qu'elle allait passer l'année à venir entre l'université d'Alger et Annaba, (...), comme si saint Augustin, à l'oeuvre duquel il venait de consacrer un an de sa vie, ne méritait plus une seconde d'attention supplémentaire. 》 (FERRARI, 2012: 90)

Dans cette situation, Libero suit le même trajet que Matthieu, une telle démarche qui l'oblige à quitter ses études, à gérer ce café maudit situé en Corse et à passer par les mêmes étapes destructives (violence, sexe, alcoolisme etc.). Si Aurélie, cette jeune fille, décide de se dépayser pour étudier l'archéologie, Libero n'est pas prêt à accomplir le moindre effort pour continuer ses études philosophiques sur Leibniz. En abandonnant ses études, il a voulu imiter Matthieu qui en avait assez de la philosophie pessimiste de Saint-Augustin, lequel avançait des maximes sur la vanité du monde ainsi que sur la faiblesse de la condition humaine:

"Peut-être songeait-il avec nostalgie à ses années d'études, aux textes qu'il avait voulu brûler sur l'autel de la stupidité du monde et dont les échos lui parvenaient pourtant encore.

Car Dieu n'a fait pour toi qu'un monde périssable, et tu es toi-même promis à la mort. " (FERRARI, 2012: 188) 
Si Matthieu renonce à ses études philosophiques, les termes de SaintAugustin demeurent inoubliables. Mais plutôt que de provoquer chez lui une tendance à la spiritualité et à l'ascétisme, ces sermons l'ont rendu de plus en plus lâche, avide de plaisir et prêt à tout pour jouir de ce monde. Comme Matthieu vit dans un univers aléatoire et que lui (en tant qu'être humain) est éphémère, cet anti-héros a décidé de profiter de chaque instant de son existence. Il a bien appris qu'il ne faut rien attendre de personne et qu'il ne doit jamais solliciter ni le secours d'un père mortel (Jacques) ni l'aide d'un père céleste:

"(...) il déposa un baiser sur le crucifix avec une piété qu'il aurait voulu ne pas feindre mais ni son père ni Dieu ne l'attendaient dans la croix, et il ne sentit rien d'autre que le contact du métal froid sur ses lèvres. ” (FERRARI, 2012:153)

Ce paragraphe qui clôt notre étude critique sert à justifier l'attitude hédoniste et épicurienne de Matthieu ou de l'anti-héros en général. D'après cet archétype, il ne faut se fier qu'aux choses concrètes et provisoires. Quant aux valeurs sublimes et absolues, elles ne relèvent que d'un monde invisible, lointain et inaccessible aux êtres humains. Dorénavant, nonchalance, lâcheté et égoïsme deviendront les seules armes capables de défendre l'anti-héros et de protéger son bonheur ici-bas. Ce type médiocre proscrit de son dictionnaire l' " au-delà » car dès le début, nous avons égard à un personnage qui ne s'intéresse point à son avenir, qu'il soit terrestre (le succès social et matériel) ou céleste (la vie éternelle).

\section{Conclusion.}

Nous avons donc l'impression que l'anti-héros abolit le fossé entre le monde et sa représentation. Quoiqu'il manque de virilité, cet archétype sert à refléter tous les dérèglements de l'époque moderne: perte des repères, dévalorisation des valeurs, interrogations métaphysiques infinies, scepticisme, sentiment permanent de déréliction etc.

L'approche dialectique que nous avons utilisée nous invite à percevoir le triomphe relatif réalisé par l'anti-héros, comme si Jérôme Ferrari avait voulu être juste en jugeant ce personnage-type. Au-delà de l'échec dans lequel il sombre, cet anti-héros est capable de réaliser certains succès éphémères. Mais si ce personnage dispose de quelques qualités exceptionnelles, il sombrera tôt ou tard dans la médiocrité. Même les solutions qu'il trouve pour mettre un terme à sa crise ne font qu'aggraver son cas. Pour lui, la chute et la défaite sont inévitables car l'homme (ou le monde en général) n'est créé que pour périr, pour être anéanti. Résignation, impuissance et soumission sont donc les attributs essentiels propres à chaque être humain. Quant au courage et à la persévérance, ce ne sont que des vertus illusoires qui ne prolongeront guère l'existence de l'être. À quoi bon lutter pour être fort et pour avoir une vie 
originale si l'on va tôt ou tard mourir et connaître une fin analogue à tous les êtres? À quoi bon chercher à modifier un univers périssable, un monde qui obéit à l'éternel retour du même?

Cette méthode dialectique acquiert aussi un aspect inductif et nous invite à émettre un jugement général: que tous les anti-héros sont d'une originalité plus touchante et plus crédible que celle des héros classiques, virils, supérieurs et puissants mais qui, en même temps, sont dépourvus de tout réalisme et de toute vraisemblance. Dans le roman de Ferrari, si Marcel et Matthieu sont considérés comme deux figures fictives, ils sont théoriquement possibles dans notre monde actuel et réel.

Dorénavant, nous ne devrons pas accuser l'anti-héros de platitude puisqu'il contribue à la grandeur du roman. En un mot, il dote l'art romanesque d'un aspect réaliste. Par contre, le héros épique (à l'instar des trois mousquetaires d'Alexandre Dumas, par exemple) n'est qu'un insulaire resté jusqu'à la fin de ses jours à l'écart de la réalité. Comme il fait preuve d'un idéal chevaleresque, ce héros incarne un absolu qui n'est pas de ce monde. Nous avons donc égard à un intrus, à un type vide et incapable de nous communiquer la douleur du monde ou la cruauté des hommes. C'est ce héros mythique qui appauvrit donc la littérature et qui marque son échec car il fait de l'écrivain un inventeur de chimères.

Bref, le fait de mépriser l'anti-héros et de glorifier les actions extraordinaires et les sentiments élevés du héros classique relève d'une myopie intellectuelle et esthétique. En effet, le roman moderne ne vit que de la mort du personnage; l'échec le plus lamentable et l'extrême défaite du personnage font le succès du Sermon sur la chute de Rome qui devient, dans ce cas, le meilleur reflet de l'angoisse existentielle.

\section{Le Corpus:}

\section{Bibliographie}

FERRARI, Jérôme (2012). Le sermon sur la chute de Rome. France. Actes Sud.

\section{I) Ouvrages consacrés à la narratologie:}

-BERTHELOT, Francis (1997). Le corps du héros. Pour une sémiologie de l'incarnation romanesque. Paris. Nathan.

- ERMAN, Michel (2006). Poétique du personnage du roman. Paris. Marketing SA.

-GLAUDES, Pierre \& al. (1991). Personnage et Histoire Littéraire. Presses Universitaires du Mirail.

- JOUVE, Vincent (2001). L'effet-personnage dans le roman. France. Presses Universitaires de France.

-MIRAUX, Jean-Philippe (1997). Le personnage de roman. Genèse, continuité, rupture. Paris. Nathan.

- ZÉRAFFA, Michel (1971). Roman et société. Paris. PUF. 
II) Ouvrages généraux:

1. Ouvrages consacrés à la critique littéraire:

-ROUAUD, Jean (2011). Le roman $d u X X$ e. France. Gallimard.

-STALLONI, Yves (2006). Dictionnaire du roman. France. Armand Colin.

- MitTerAND, Henri (1980). Le discours du roman. Paris. Presses Universitaires de France.

\section{Ouvrage portant sur la mythocritique:}

- ELIADE, Mircea (1969). Le mythe de l'éternel retour. Archétypes et répétition. France. Gallimard.

\section{Résumé de la recherche}

Dans cette recherche, nous parlerons d'un archétype très célèbre dans la littérature moderne: « l'anti-héros » qui se voit dépouillé de toute positivité. À travers une étude approfondie du Sermon sur la chute de Rome de Jérôme Ferrari, nous analyserons la psychologie de l'anti-héros, incarné par Marcel Antonetti. Dès son bas âge, ce personnage souffre d'un ulcère gastralgique et se croit capable de lutter contre ce démon, à la façon d'un héros épique. Mais avec le temps, Marcel doit s'enliser dans la médiocrité: il commence à avouer que tous les efforts déployés pour combattre la maladie et la mort (ou la fatalité en général) s'avèrent vains et stériles. À cette détérioration physique s'ajoute l'échec professionnel. Au cours de sa vieillesse, Marcel doit reconnaître sa défaite totale vis-à-vis du destin. Cependant, en dépit de la mort prochaine et inévitable de ce personnage, l'archétype de « l'anti-héros » demeure vivant et éternel dans l'oeuvre de Ferrari, et ce grâce à Matthieu Antonetti, le petit-fils de Marcel, qui a hérité des défauts de son grand-père: solitude, rêverie, médiocrité etc. Nous avons donc égard à une nouvelle épave.

Notre problématique consiste alors à prouver que l'anti-héros est plus crédible que le héros classique, cet être chimérique qui n'existe que dans un monde utopique. En effet, l'anti-héros sert à nourrir la littérature et à la doter d'un aspect vraisemblable et réaliste, alors que le héros aux qualités extraordinaires met en doute la crédibilité de l'écrivain. Grâce à la méthode « dialectique », nous aborderons une thèse qui fait de Marcel un héros " positif » apte à combattre la maladie. Ensuite, nous examinerons l'anti-thèse qui focalise sur la condition lamentable de ce personnage (son dépaysement au sein de ce monde hostile, ses tares et ses défauts: lâcheté, infériorité et impuissance).

Pour ce qui est de la conclusion, elle consiste à reconnaître que cet archétype (l'anti-héros) est immortel et que c'est lui qui nourrit l'oeuvre littéraire et qui la dote d'un aspect crédible.

Les mots-clés: l'anti-héros- l'individu et la société- l'éternel retour- le nihilisme- le sentiment d'infériorité - l'hostilité du monde.
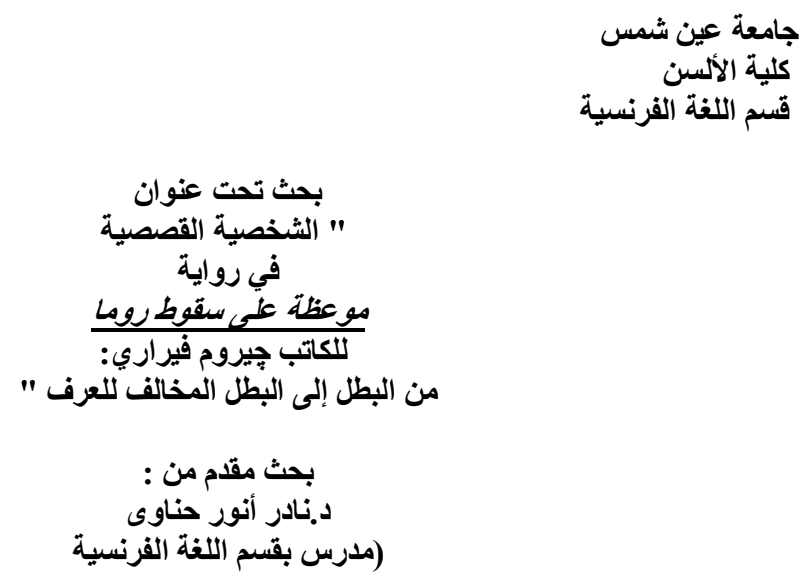


$$
\text { كلية الألسن }
$$

\section{ملخص البحث باللغة العربية \\ عنوان البحث:}

\section{الشخصية القصصية في رواية "موعظة على سقوط روما" \\ للكاتب جيروم فيراري: \\ من البطل إلى البطل المخالف للعرف}

في هذا البحث، يتحدث الباحث عن نمط مختلف في الأدب الحديث ألا وهو "البطل المخالف للعرف" الذي لا يتمتع

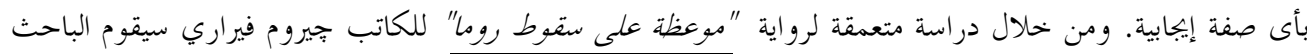

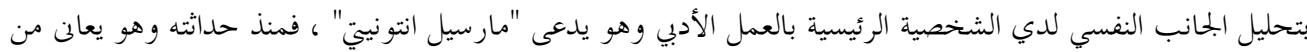

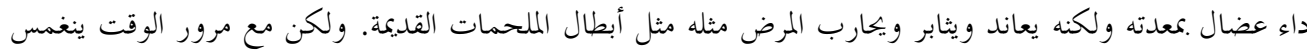

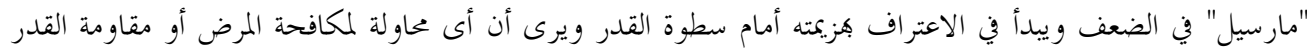

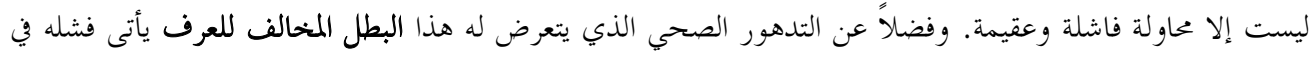

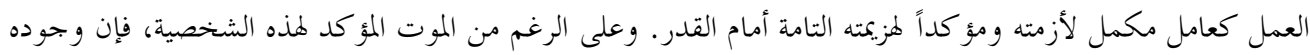

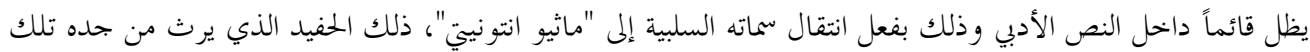
اللعنة الثى تحوله إلى شخصية حدية جديدة ومعادية للمجتمع.

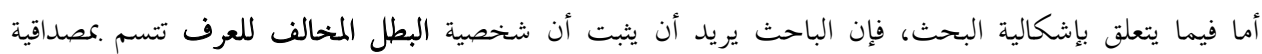

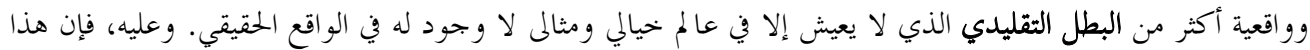

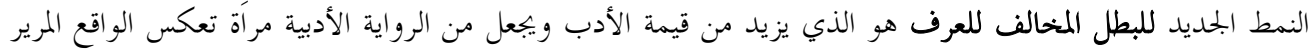

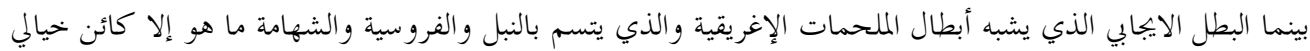

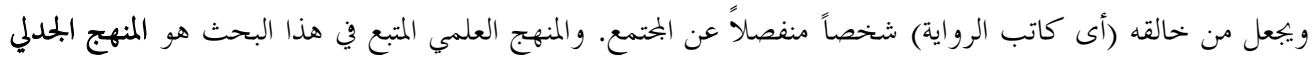

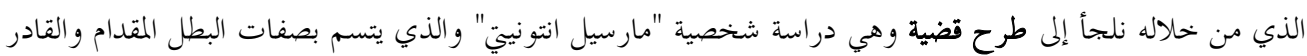

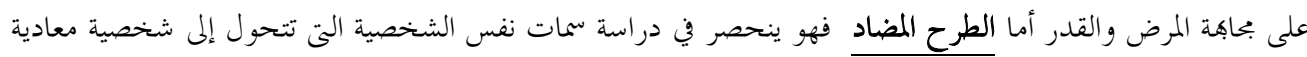

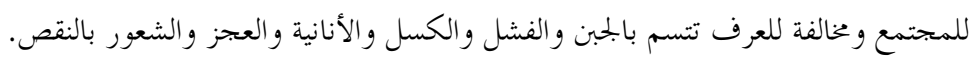
وفي خاتمة البحث، يريد الباحث أن يضيف أن البطل المخالف للعرف ما هو إلا نتاج للمجتمع الذي نعيش فيه، كما

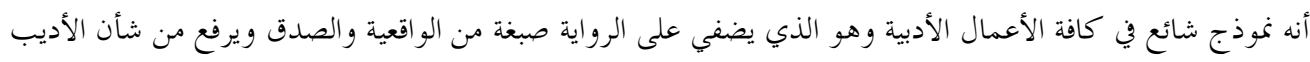
ومن قيمة الأدب. 